\title{
Development of Ferroelectric RAM (FRAM) for Mass
}

\section{Production}

Takashi Eshita ${ }^{1}$, Wensheng Wang ${ }^{1}$, Kou Nakamura ${ }^{1}$, Souichirou Ozawa ${ }^{1}$, Youichi Okita ${ }^{1}$, Satoru Mihara ${ }^{1}$, Yukinobu Hikosaka ${ }^{1}$, Hitoshi Saito ${ }^{1}$, Junichi Watanabe ${ }^{1}$, Ken'ichi Inoue ${ }^{1}$, Hideshi Yamaguchi ${ }^{2}$ and Kenji Nomura $^{2}$

1. System Memory Business Division, Fujitsu Semiconductor Limited, Kohoku-ku, Yokoham 222-0033, Japan

2. Factory Engineering Laboratory, Fujitsu Laboratories Limited, 10-1 Morinosato-Wakamiya, Atsugi, Kanagawa 243-0197, Japan

\begin{abstract}
: we have developed ferroelectric capacitor fabrication technique to realize low-voltage and high-density ferroelectric random access memory (FRAM). High temperature deposited $\mathrm{IrO}_{x}$ top electrode reveals high crystalline quality which drastically reduces the degradation of ferroelectric film by preventing hydrogen diffusion into ferroelectric film. This improvement enables us to commercialize highly-reliable 1T1C FRAM with memory density of $4 \mathrm{Mb}$ or larger.
\end{abstract}

Key words: Ferroelectric, PZT, LCSPZT, IrO.

\section{Introduction}

Ferroelectric random access memory (FRAM) is the first commercialized memory among advanced non-volatile memories such as magnetoresistive RAM (MRAM), phase change RAM (PCRAM), and resistive RAM (ReRAM) [1-4]. FRAM has been reported to have advantages of lower power consumption and higher switching endurance in comparison with commercial base FLASH memory, Spin-Torque-Transfer (STT) MRAM, and PCRAM [5]. We have developed fabrication technologies focusing on reliability, decreasing operation voltage, and power consumption. In our development, an interface control between ferroelectric material and electrode is found to be crucial for decreasing the operation voltages with maintaining the large polarization. This capacitor fabrication improvement with circuit improvements of the sensing amplifier [6, 7] has successfully realized robust reliability of the $4 \mathrm{Mb}$ or larger memory-size FRAM. Our FRAM has evolved from $5 \mathrm{~V}$ operation and $0.5 \mu \mathrm{m}$ node CMOS to $1.8 \mathrm{~V}$ operation and 0.18

Corresponding author: Takashi Eshita, research field: semiconductor production development. E-mail: eshita@jp.fujitsu.com. $\mu \mathrm{m}$ node CMOS. This paper describes the key technologies of FRAM fabrication process and circuit design.

\section{Experiments}

FRAM fabrication process consists of conventional $0.18 \mu \mathrm{m}$ CMOS and ferroelectric capacitor fabrication processes which were described elsewhere [3]. $\mathrm{IrO}_{x}$ top electrode, Pt bottom electrode and LCSPZT ( $\mathrm{La}, \mathrm{Sr}$ and $\mathrm{Ca}$ doped PZT) were deposited by sputtering. Fabrication procedures of conventional and new ferroelectric capacitor are shown in Fig. 1. We employed two-step annealing process consisting of anneal 1 (about $650{ }^{\circ} \mathrm{C}$ ) and anneal 2 (about $750{ }^{\circ} \mathrm{C}$ ) by using rapid thermal annealing (RTA) to obtain good crystalline LCSPZT with uniform sized grain. Both of those two annealing procedures are carried out before depositing the top electrode. While top electrode $\mathrm{IrO}_{x}(x$ is around 2.0) deposited at room temperature in the conventional procedure, the 1 st top electrode $\mathrm{IrO}_{y}(y$ is around 1.9) about $350^{\circ} \mathrm{C}$, and then the 2 nd top electrode $\mathrm{IrO}_{x}$ was deposited at room temperature in the new capacitor fabrication procedure. Oxidation stages of $x$ and $y$ were measured by the 2 nd ion mass spectroscopy. 


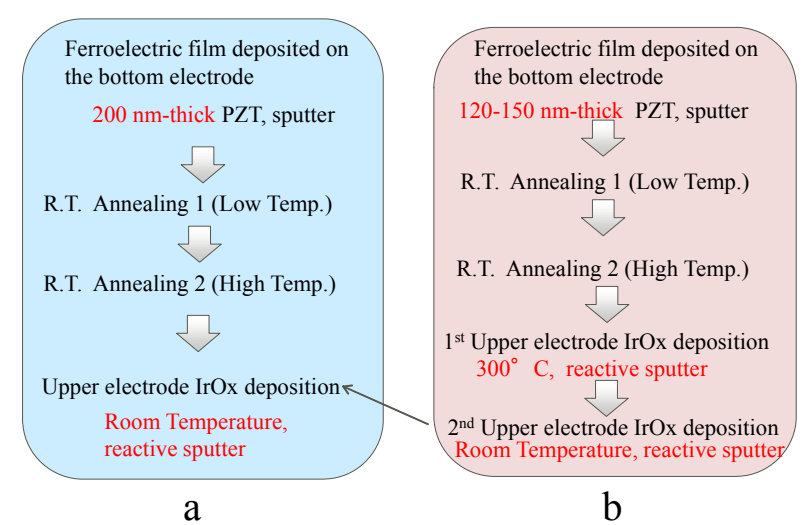

Fig. 1 (a) Fabrication procedures of conventional; (b) new developed ferroelectric capacitor.

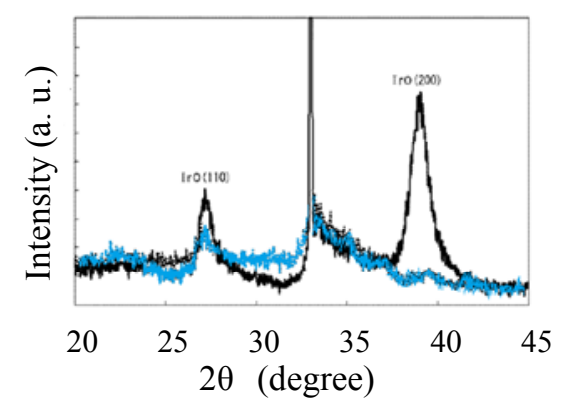

Fig. 2 X-ray diffraction patterns of conventional $\operatorname{IrO}_{x}$ (blue line) and new (black line) $\mathrm{IrO}_{y}$.

\section{Results and Discussion}

Although we tried to reduce the thickness of the ferroelectric film from $200 \mathrm{~nm}$ to $150 \mathrm{~nm}$ to obtain 1.8 $\mathrm{V}$ operation ferroelectric capacitor by conventional procedure, ferroelectric capacitor with $150 \mathrm{~nm}$ thick LCSPZT had poor ferroelectric properties, such as small switching polarization $\left(Q_{s w}\right)$ and large coercive voltage $\left(V_{c}\right)$, which prevents the FRAM from reading and rewriting operations at $1.8 \mathrm{~V}$. Since we found the interface control between the $\mathrm{IrO}_{x}$ top electrode and PZT is important to lower the operation voltage, we improved the top electrode deposition process. We have found that high temperature about $300{ }^{\circ} \mathrm{C}$ deposition of $\mathrm{IrO}_{x}$ drastically improved the crystal quality, as shown in Fig. 2. Fig. 3 shows the cross-sectional TEM images of the conventional and new ferroelectric capacitors. It is clear that $\mathrm{IrO}_{x}$ near the interface of new capacitor is very smooth. Improvements of the $Q_{s w}$ and the $V_{c}$ are also clear as shown in Fig. 4. Leakage current of the new ferroelectric capacitor is slightly larger than that of conventional capacitor, as shown in Fig. 5, however, which does not significantly influence to the FRAM function. Relatively larger leakage current of small capacitor may be due to its large side area of the capacitor. Our reliability examination results of switching endurance and imprint endurance (opposite state (OS) rate) are shown in Fig. 6. In the OS rate, the closer value to zero indicates the better imprint endurance. Reliability of FRAM with new ferroelectric capacitor is clearly superior to that of conventional FRAM.

The interface region of ferroelectric film near the top electrode is degraded by inter-reaction during the heat treatments in metallization, thus resulting in the

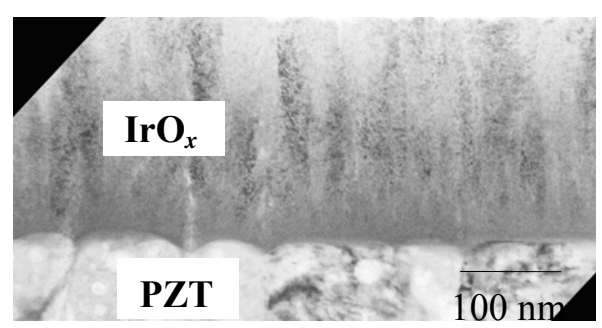

(a)

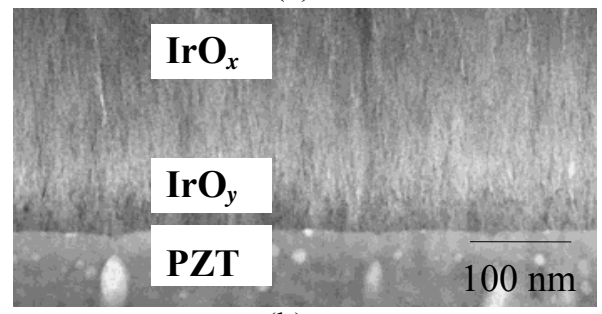

(b)

Fig. 3 Cross-sectional TEM micrograph of the ferroelectric capacitors. (a) Conventional; (b) new capacitor. Oxidation state as denoted $x$ and $y$ are about 1.9 and 1.8, respectively.
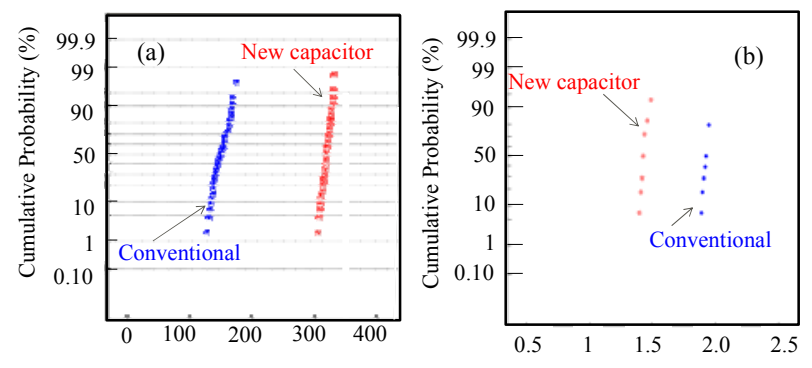

Switching Polarization (fC/cell) Coercive Voltage (V)

Fig. 4 In-wafer distribution of switching polarization at (a) $1.8 \mathrm{~V}$ and (b) coercive voltage. 


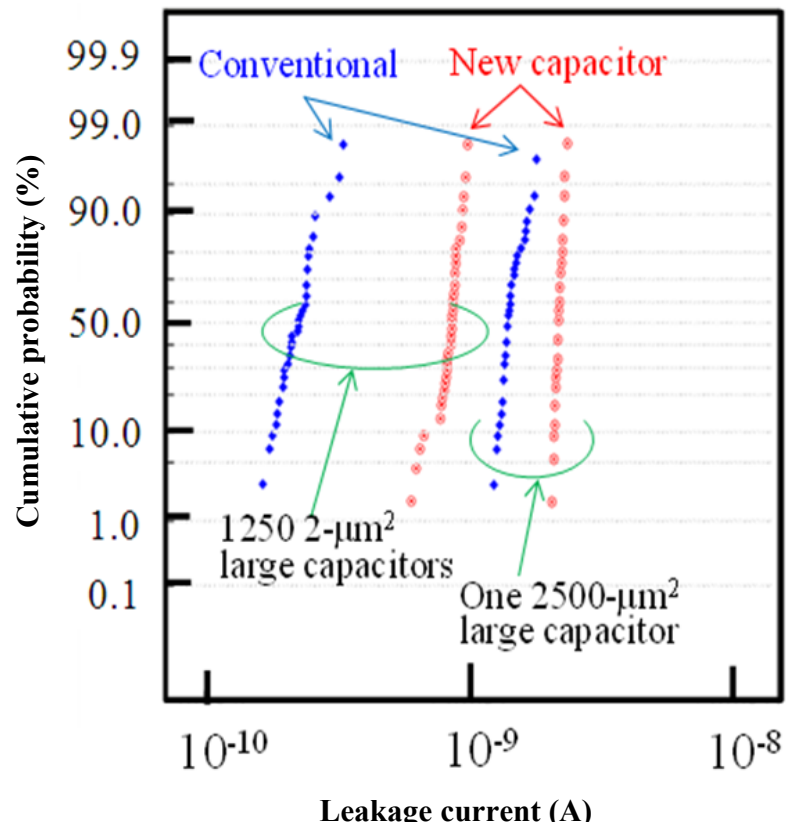

Fig. 5 Leakage currents distribution on a wafer for monitors consisting of (a) $1,250 \times 2 \mu^{2}$ large capacitors and (b) one $2,500 \mu \mathrm{m}^{2}$ large capacitor.

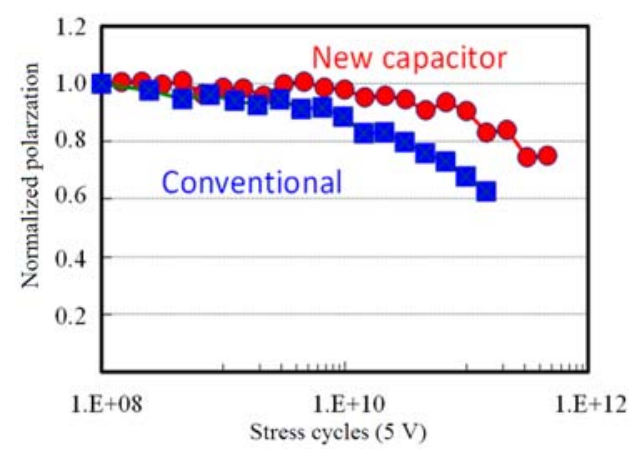

(a)

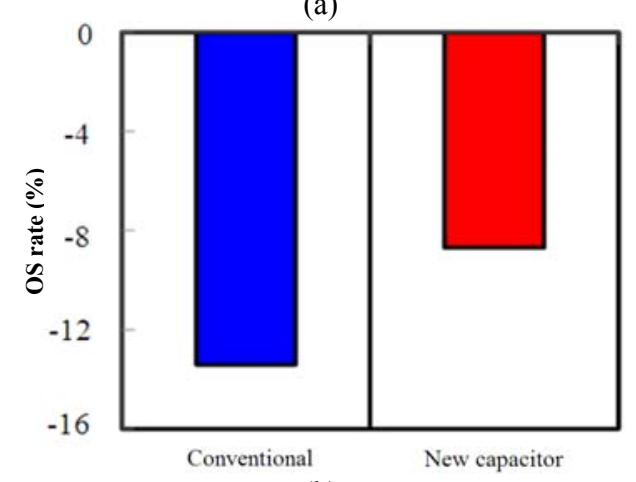

(b)

Fig. 6 Reliability examination results of (a) switching endurance and (b) imprint endurance or OS rate.

degradation of ferroelectric properties. Since the thickness of the degraded region does not change unless employing the same fabrication procedure, degradation

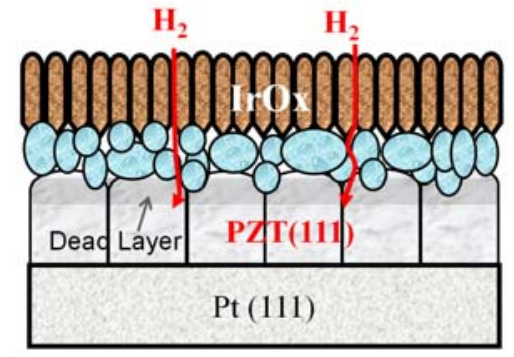

(a)

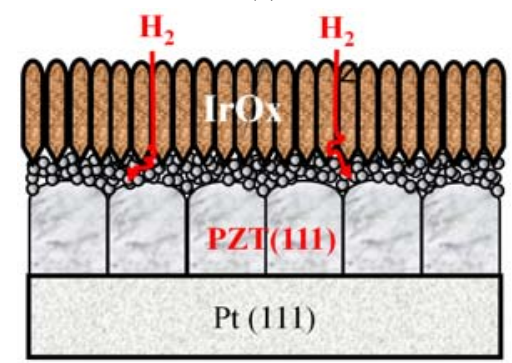

(b)

Fig. 7 Schematic cross-sectional ferroelectric capacitors, (a) conventional and (b) new capacitor.

of the ferroelectric properties is more sever for thin ferroelectric film. In the conventional capacitor, above mentioned degraded region of ferroelectric film possibly enlarged by large inter reaction because as-deposited $\mathrm{IrO}_{x}$ is initially amorphous and its diffusion speed is expected to be large. After heat treatment, while the upper portion of the top electrode found to be composed of columnar-shaped grain, the lower portion is composed of round-shaped large grain from precise TEM observation, as schematically shown in Fig. 7a, which may be due to large inter diffusion. Since XRD analysis has proven that $\mathrm{IrO}_{x}$ contains metallic Ir, it is possible that hydrogen generated during the metallization catalytically activated by the metallic $\mathrm{Ir}$ reduces $\mathrm{IrO}_{x}$ by itself and the ferroelectric film. When the ferroelectric film is reduced, characteristics of the ferroelectric capacitor are degraded [8]. It is also considered that oxygen vacancies in the $\mathrm{IrO}_{x}$ cause much easier hydrogen diffusion. In the new ferroelectric capacitor fabrication procedure, since precise SEM and TEM observations revealed the as-deposited $\mathrm{IrO}_{y}$ is composed of very fine grain, as schematically depicted in Fig. 7b, it is supposed to be much more 
difficult to react with ferroelectric film, as suggested by smoother interface in Fig. 3b, which possibly prevents interface degrading layer from enlarging and also prevents hydrogen from diffusing into ferroelectric film. Hence, we have successfully developed $1.8 \mathrm{~V}$ operable 1T1C $4 \mathrm{Mb}$ FRAM with excellent reliability.

\section{Conclusions}

We have developed new ferroelectric capacitor fabrication technology to realize a mass production of $1.8 \mathrm{~V}$ operation 1T1C 4Mb FRAM. Superior characteristics and highly reliable ferroelectric capacitor was obtained by employing high temperature $\mathrm{IrO}_{y}$ top electrode deposition.

\section{Acknowledgments}

The authors wish to express their appreciation to Mr. Amane Inoue and Mr. Masato Matsumiya for their valuable cooperation.

\section{References}

[1] Evans, J. T., and Womack, R. 1988. "An Experimental 512-Bit Non-volatile Memory with Ferroelectric Storage Cell.” IEEE J. Solid-St. Circ. 23: 1171-5.

[2] Eaton, S. S., Butler, D. B., Parris, M., Wilson, D., and McNeillie, H. 1988. "A Ferroelectric Non-volatile Memory.” Presented at IEEE Int. Solid-State Circuit Conf.
Dig. Tech. Paper, San Francisco CA, 130-1.

[3] Yamazaki, T., Inoue, K., Miyazawa, H., Nakamura, M., Sashida, N., Satomi, R., Kerry, A., Katoh, Y., Noshiro, H., Takai, K., Shinohara, R., Ohno, C., Nakajima, T., Furumura Y., and Kawamura, S. 1997. "Advanced $0.5 \mu \mathrm{m}$ FRAM Device Technology with Full Compatibility of Half-Micron CMOS Logic Device." Presented at IEEE Int. Electron. Devices Meeting Tech. Dig., Washinton DC, 613-6.

[4] Moise, T. S., Summerfelt, S. R., Xing, G., Colombo, L., Sakoda, T., Gilbert, S. R., Loke, A., Ma, S., Kavari, R., Wills, L. A., and Hsu, T. 1999. "Electrical Properties of Submicron $(0.13 \mu \mathrm{m}) \mathrm{Ir} / \mathrm{PZT} / \mathrm{Ir}$ Capacitors Formed on W Plugs.” Presented at IEEE Int. Electron Device Meeting Tech. Dig., Washinton DC, 940-2.

[5] International Technology Roadmaps for Semiconductor. 2011. "Emerging Research Device, 2011ERD3." Accessed July 21, 2014. http://www.itrs.net/Links/2011ITRS/Home2011.Htm.

[6] Kawashima, S., Fukushi, I., Morita, K., Nakabayashi, K., Nakazawa, M., Yamane, K., Hirayama, T., and Endo, T. 2007. "A Reliable 1T1C FeRAM Using a Thermal History Tracking 2T2C Dual Reference Level Technique for a Smartcard Application Chip." IEICE Transaction E90-C 1941-8.

[7] Kawashima, S., Endo, T., Yamamoto, A., Nakabayashi, K., Nakazawa, M., Morita, K., and Aoki, M. 2002. "Bitline GND Sensing Technique for Low-Voltage Operation FeRAM.” IEEE J. Solid-State Circuits. 37: 592-8.

[8] Cross, J. S., Horii, Y., Mizuta, N., Watanabe, S., Eshita, T., and Aoki, M. 2002. " $\mathrm{IrO}_{2} / \mathrm{Pb}(\mathrm{Zr}, \mathrm{Ti}) \mathrm{O}_{3} / \mathrm{Pt}$ Capacitor Degradation with $\mathrm{D}_{2}$ Gas at Elevated Temperature." Jpn. J. Appl. Phys. 41: 698-701. 